\title{
Universal features of Lifshitz Green's functions from holography
}

\author{
Cynthia Keeler, ${ }^{a}$ Gino Knodel, ${ }^{b}$ James T. Liu $^{b}$ and Kai Sun ${ }^{b}$ \\ ${ }^{a}$ Niels Bohr International Academy, Niels Bohr Institute, University of Copenhagen, \\ Blegdamsvej 17, DK 2100, Copenhagen, Denmark \\ ${ }^{b}$ Michigan Center for Theoretical Physics, Randall Laboratory of Physics, \\ The University of Michigan, Ann Arbor, MI 48109-1040, U.S.A. \\ E-mail: keeler@nbi.ku.dk, gknodel@umich.edu, jimliu@umich.edu, \\ sunkai@umich.edu
}

ABSTRACT: We examine the behavior of the retarded Green's function in theories with Lifshitz scaling symmetry, both through dual gravitational models and a direct field theory approach. In contrast with the case of a relativistic CFT, where the Green's function is fixed (up to normalization) by symmetry, the generic Lifshitz Green's function can a priori depend on an arbitrary function $\mathcal{G}(\hat{\omega})$, where $\hat{\omega}=\omega /|\vec{k}|^{z}$ is the scale-invariant ratio of frequency to wavenumber, with dynamical exponent $z$. Nevertheless, we demonstrate that the imaginary part of the retarded Green's function (i.e. the spectral function) of scalar operators is exponentially suppressed in a window of frequencies near zero. This behavior is universal in all Lifshitz theories without additional constraining symmetries. On the gravity side, this result is robust against higher derivative corrections, while on the field theory side we present two $z=2$ examples where the exponential suppression arises from summing the perturbative expansion to infinite order.

KEYwords: Holography and condensed matter physics (AdS/CMT), AdS-CFT Correspondence

ArXiv EPrint: 1505.07830 


\section{Contents}

1 Introduction 1

2 The Green's function in a scale invariant theory 3

3 Holographic Lifshitz models 4

3.1 Bulk higher derivatives 6

$\begin{array}{lll}3.2 & \text { Consistency of the higher derivative expansion } & 7\end{array}$

$\begin{array}{llr}4 & \text { WKB analysis of the spectral function } & 8\end{array}$

$5 \quad$ Field theory models with $z=2 \quad 14$

$\begin{array}{ll}\text { 5.1 The quadratic band crossing model } & 14\end{array}$

$\begin{array}{lll}\text { 5.1.1 Renormalization group analysis } & 16\end{array}$

$\begin{array}{ll}\text { 5.1.2 Boson correlation functions } & 16\end{array}$

$\begin{array}{lll}\text { 5.1.3 Dirac theory revisited and systems with higher } z & 18\end{array}$

$\begin{array}{ll}\text { 5.1.4 Limitations of the analysis } & 19\end{array}$

$\begin{array}{ll}5.2 \text { The quantum Lifshitz model } & 19\end{array}$

6 Discussion $\quad 21$

$\begin{array}{ll}\text { A Perturbative expansion of the WKB integral } & 23\end{array}$

B The free quadratic band crossing theory $\quad 24$

\section{Introduction}

The AdS/CFT correspondence provides us with a remarkable strong-weak coupling duality between a bulk gravitational theory and a boundary field theory in one fewer dimension, and as such it has found numerous applications in calculating interesting observables of strongly coupled field theories using weakly coupled holographic methods. In recent years, the number of proposed gravity duals to interesting strongly coupled QFTs has increased significantly. In addition to the relativistic case of asymptotically AdS backgrounds, there exist interesting gravitational duals exhibiting non-relativistic (or Lifshitz-) scaling symmetry [1], as well as Schrödinger symmetry [2-4]. These non-relativistic backgrounds are particularly relevant for studying condensed matter systems at strong coupling, where exact analytic results are difficult to obtain using traditional methods.

In many standard cases, the mapping between bulk and boundary is easily obtained. This is especially true when there is a brane interpretation, such as the familiar picture of IIB theory on $\mathrm{AdS}_{5} \times S^{5}$. In this case, the $\mathrm{AdS}_{5}$ supergroup $\mathrm{SU}(2,2 \mid 4)$ is identical 
to the superconformal symmetry group of the four-dimensional $\mathcal{N}=4$ super-Yang Mills theory. As a result, all observables are constrained by the superconformal symmetry, and in particular the two-point functions are fully determined up to normalization. For example, the retarded scalar Green's function in momentum space must have the form

$$
G_{R}\left(q^{2}\right)=A\left(-q^{2}\right)^{\Delta-2}, \quad q^{2}=\omega^{2}-|\vec{k}|^{2},
$$

where $A$ is an overall constant and $\Delta$ is the conformal dimension of the scalar operator $\mathcal{O}_{\Delta}$.

The mapping between condensed matter systems and backgrounds with non-relativistic scaling symmetry is often less obvious. In this case, we must often fall back to the general strategy of constructing a holographic dual to a given field theory by matching symmetries and conserved quantities [1-4]. Moreover, non-relativistic scale invariance is no longer sufficient to fully constrain the form of the two-point functions. Consider, for example, the case of Lifshitz scaling with dynamical exponent $z$, where energy and momentum scale as $\omega \rightarrow \lambda^{z} \omega$ and $\vec{k} \rightarrow \lambda \vec{k}$, respectively. This scaling symmetry only constrains the form of the Green's function up to an arbitrary function of the scale-invariant quantity $\hat{\omega}=\omega /|\vec{k}|^{z}$ :

$$
G_{R}(\omega, \vec{k})=|\vec{k}|^{2 \nu z} \mathcal{G}(\hat{\omega})
$$

Here $\nu$ is the energy scaling dimension, and the momentum-dependent prefactor is chosen to give $G_{R}$ the proper scaling dimension.

The form of the Green's function (1.2) holds for any (isotropic) scale-invariant theory, whether computed directly from the field theory or via the holographic dual. However, in general, $\mathcal{G}(\hat{\omega})$ cannot be fixed by matching symmetries alone. (If additional symmetries are imposed, such as $z=2$ Schrödinger symmetry, then the Green's function may become fully determined.) This suggests that symmetries are not sufficient for connecting nonrelativistic theories to their holographic duals, and in particular that the duality map must include additional dynamical information.

At the same time, the bulk theory yields a preferred choice of the Green's function obtained from the classical two-derivative bulk action. For $z=2$ Lifshitz, the holographic scalar Green's function was obtained analytically in [1], while a WKB calculation for arbitrary $z>1$ demonstrated a characteristic exponential suppression of the spectral weight (i.e. the imaginary part of the Green's function) in the limit $\hat{\omega} \rightarrow 0$ [5]. This has been interpreted as an "insensitivity" of the boundary theory to small changes of the geometry near the horizon. The same exponential behavior is responsible for making the smearing function of both Schwarzschild-AdS and Lifshitz spacetime a distribution rather than a true function [6,7], and has been interpreted as a loss of bulk locality for such non-relativistic geometries [8].

It is natural to expect that different field theoretic models with the same dynamical exponent $z$ will yield different Green's functions. This raises the issue as to how the holographic dual can distinguish among these models. For unbroken scaling symmetry, the bulk geometry is essentially fixed to be pure Lifshitz (if we work within the context of general relativity; see e.g. [9-11] for other approaches). Thus the background alone cannot distinguish between different models, and we are mainly left with the dynamics of the bulk 
fields as the distinguishing characteristic. In particular, the addition of higher derivative terms to the bulk equations of motion will directly affect the form of the holographic Green's function. This is in contrast with the relativistic case, where higher derivative corrections may affect the constant $A$ in (1.1), but will not otherwise modify the functional form of the retarded Green's function.

Once we allow for a higher derivative expansion in the bulk, it may seem that some predictive power is lost, since the holographic Green's function would in principle be sensitive to all of the infinitely many higher derivative terms. However, we demonstrate that there are universal features that remain. In particular, the characteristic exponential suppression of the spectral function in the low frequency regime found in [5] is robust with respect to higher derivatives in the bulk, as long as the frequencies stay above a (momentumdependent) cutoff.

We furthermore show that this exponential suppression arises in field theory models with $z=2$ scaling. In particular, for both the quadratic band crossing model of [12] and the quantum Lifshitz model [13], a simple kinematical argument demonstrates that the exponential suppression arises because one has to go to higher and higher orders in the perturbative expansion to see non-zero spectral weight in the limit $\hat{\omega} \rightarrow 0$.

This paper is organized as follows. In section 2, we briefly review the form of the scalar Green's function in a theory with Lifshitz scaling. Then in section 3, we set up the computation of the Green's function in a holographic Lifshitz model with bulk higher derivatives. Following that, we perform a WKB analysis of the spectral function in section 4 and show that it has a universal exponential suppression at small $\hat{\omega}$. In section 5, we demonstrate that this exponential suppression can be seen directly from a field theory perspective. We focus on the quadratic band crossing model, but also consider the quantum Lifshitz model. Finally, we conclude in section 6 with a conjecture that this suppression is universal for all Lifshitz theories in the absence of further constraining symmetries.

\section{The Green's function in a scale invariant theory}

In a translationally invariant theory, the retarded Green's function is naturally written in momentum space as $G_{R}(\omega, \vec{k})$. Furthermore, unitarity and causality demand that $G_{R}$ is analytic in the upper half of the complex $\omega$-plane. For a scale-invariant theory, the conditions on the Green's function are much stronger. In particular, for Lifshitz scaling symmetry with dynamical exponent $z$

$$
\vec{x} \rightarrow \Lambda \vec{x}, \quad t \rightarrow \Lambda^{z} t
$$

scale and rotational invariance demand that $G_{R}$ cannot depend on $\omega$ and $\vec{k}$ separately, but must have the form

$$
G_{R}(\omega, \vec{k})=|\vec{k}|^{2 \nu z} \mathcal{G}(\hat{\omega}) \quad \text { where } \quad \hat{\omega} \equiv \frac{\omega}{|\vec{k}|^{z}}
$$

Here $\nu$ is the energy scaling dimension and $\mathcal{G}(\hat{\omega})$ is analytic in the upper half $\hat{\omega}$ plane. 
Non-relativistic scale invariance by itself does not further constrain the form of $\mathcal{G}(\hat{\omega})$. However, additional symmetries can fix it completely. For example, relativistic conformal invariance (for the case $z=1$ ) constrains $G_{R} \sim\left(-q^{2}\right)^{\nu}$ where $q^{2}=-k_{\mu} k^{\mu}=\omega^{2}-|\vec{k}|^{2}$. This is equivalent to taking the function

$$
\mathcal{G}_{\mathrm{CFT}}=A\left(1-\hat{\omega}^{2}\right)^{\nu}
$$

where $A$ is a constant. Similarly, full Schrödinger symmetry (for $z=2$ ) [2-4] requires

$$
\mathcal{G}_{\text {Sch }}=A(1-2 m \hat{\omega})^{2 \nu},
$$

where $A$ is again a constant, and $m$ is the eigenvalue of the mass-operator of the Schrödinger algebra.

While the relativistic and Schrödinger cases are the most extensively studied, we are mainly interested in exploring the features of the function $\mathcal{G}(\hat{\omega})$ for Lifshitz models without additional symmetries using holographic methods. In general, $\mathcal{G}$ will depend on the details of the model. However, some universal properties can be deduced in both the small and large $\hat{\omega}$ limits. For $\hat{\omega} \rightarrow 0$, the only dimensionful quantity that remains is $|\vec{k}|$. Hence $G_{R}$ must behave as $|\vec{k}|^{2 \nu z}$, or equivalently

$$
\mathcal{G}(\hat{\omega} \rightarrow 0) \sim \text { const. }
$$

On the other hand, when $\hat{\omega} \rightarrow \infty$, the dependence on $|\vec{k}|$ drops out, and we must have

$$
\mathcal{G}(\hat{\omega} \rightarrow \infty) \sim \hat{\omega}^{2 \nu}
$$

As can be seen from (2.3) and (2.4), the $z=1$ and Schrödinger $z=2$ cases both satisfy these properties.

The retarded Green's function is in general complex, and this ought to be kept in mind when considering the limiting behaviors given above. Of particular interest is the general behavior of the spectral function $\chi(\omega, \vec{k})=2 \operatorname{Im} G_{R}(\omega, \vec{k})$. For large $\omega$, the spectral function scales as $\chi \sim \omega^{2 \nu}$, consistent with (2.6), as well as the relativistic and Schrödinger cases. The small $\omega$ limit, on the other hand, is more subtle. While scaling symmetry demands $\chi \sim 2|\vec{k}|^{2 \nu z} \operatorname{Im} \mathcal{G}(\hat{\omega})$, with $\operatorname{Im} \mathcal{G}(\hat{\omega})$ approaching a constant as $\hat{\omega} \rightarrow 0$, this constant is in fact zero for the $z=1$ and $z=2$ Schrödinger cases. Moreover, for these cases $\chi$ is identically vanishing for a range of $\hat{\omega}$ near zero. However, this no longer needs to be the case in theories with Lifshitz scaling, but without additional symmetries. Nevertheless, as we have shown in [5], in the latter case the spectral function is at most exponentially small in the limit $\hat{\omega} \rightarrow 0$, at least in the two-derivative holographic theory. What we will show below is that this exponential suppression of $\chi$ remains robust, even when higher derivative corrections are included, as long as the perturbative expansion is kept under control.

\section{Holographic Lifshitz models}

The Lifshitz symmetry (2.1) can be realized in a gravitational background given by the metric

$$
d s_{d+2}^{2}=\frac{-d t^{2}+d \rho^{2}}{\rho^{2}}+\frac{d \vec{x}^{2}}{\rho^{2 / z}} .
$$


The boundary of the bulk spacetime is located at $\rho=0$, while the horizon is at $\rho=\infty$. For simplicity, we examine the scalar Green's function, which can be holographically computed from the action of a bulk scalar $\phi(t, \vec{x}, \rho)$.

At the two-derivative level, the minimally coupled equation of motion for $\phi$ is simply $\left(\square-m^{2}\right) \phi=0$. This system has been extensively studied, and the holographic computation of the retarded Green's function is by now standard [14]. Working in momentum space and taking

$$
\phi(t, \vec{x}, \rho)=e^{i(\vec{k} \cdot \vec{x}-\omega t)} \rho^{d / 2 z} \psi(\rho),
$$

we find that $\psi(\rho)$ satisfies the Schrödinger-like equation $-\psi^{\prime \prime}+U_{0} \psi=0$ where

$$
U_{0}=\frac{\nu^{2}-1 / 4}{\rho^{2}}+\frac{|\vec{k}|^{2}}{\rho^{2-2 / z}}-\omega^{2} .
$$

and

$$
\nu=\sqrt{m^{2}+\left(\frac{d+z}{2 z}\right)^{2}} .
$$

We can highlight the scaling properties of the solution by defining the dimensionless coordinate

$$
\hat{\rho}=\rho|\vec{k}|^{z} .
$$

The Schrödinger-like equation now takes the form

$$
-\psi^{\prime \prime}(\hat{\rho})+\hat{U}_{0}(\hat{\rho}) \psi(\hat{\rho})=0, \quad \hat{U}_{0}(\hat{\rho})=\frac{\nu^{2}-1 / 4}{\hat{\rho}^{2}}+\frac{1}{\hat{\rho}^{2-2 / z}}-\hat{\omega}^{2} .
$$

In order to apply the AdS/CFT prescription for calculating the retarded Green's function, we need to examine the solution near the boundary at $\hat{\rho}=0$ and as it approaches the horizon at $\hat{\rho}=\infty$. In the limit $\hat{\rho} \rightarrow 0$, the Schrödinger potential is dominated by the $\left(\nu^{2}-1 / 4\right) / \hat{\rho}^{2}$ term, and we find the boundary behavior

$$
\psi(\hat{\rho} \rightarrow 0) \sim A \hat{\rho}^{\frac{1}{2}-\nu}+B \hat{\rho}^{\frac{1}{2}+\nu} .
$$

Here we have used the convention that $B$ is the coefficient of the normalizable mode, while $A$ is the coefficient of the non-normalizable mode. For $z>1, \hat{U}_{0}$ approaches $-\hat{\omega}^{2}$ at the horizon, so the solution is oscillatory:

$$
\psi(\hat{\rho} \rightarrow \infty) \sim a e^{i \hat{\omega} \hat{\rho}}+b e^{-i \hat{\omega} \hat{\rho}}
$$

For the retarded Green's function, we take infalling boundary conditions, which correspond to setting $b=0$. In this case, we find

$$
\mathcal{G}(\hat{\omega})=\left.\frac{B}{A}\right|_{b=0},
$$

where the relation between $\{A, B\}$ at the boundary and $\{a, b\}$ at the horizon is obtained by solving the Schrödinger problem (3.6). 


\subsection{Bulk higher derivatives}

At the two-derivative level, the solution for $\mathcal{G}(\hat{\omega})$ has been extensively studied, and analytic results may be obtained for $z=1$ and $z=2[1,14]$. However, as we emphasized in section 2, scaling symmetry by itself does not fully constrain the form of the Green's function. This raises the question of where the freedom of arbitrarily choosing the function $\mathcal{G}$ arises in the holographic dual. If we work within general relativity, there are two natural possibilities: the first is the choice of background metric, and the second is the form of the scalar equation. However, the metric (3.1) is essentially unique (up to coordinate transformations) once we have imposed Lifshitz scaling. This leaves us with modification of the equation of motion.

From a bulk effective field theory point of view, it is possible to include higher derivative terms in the scalar equation. In momentum space, non-radial derivatives in the effective action show up as powers of $\omega$ and $\vec{k}$, while additional $\rho$ derivatives lead to a higher order differential equation for $\psi(\rho)$. If there are no additional $\rho$ derivatives, then the momentum space equation remains second order and can be brought into Schrödinger form just as above. This time, however, the effective Schrödinger potential in (3.6) generalizes to

$$
\hat{U}(\hat{\rho})=\frac{\nu^{2}-1 / 4}{\hat{\rho}^{2}}+\frac{1}{\hat{\rho}^{2-2 / z}}-\hat{\omega}^{2}+\frac{1}{\hat{\rho}^{2}} f\left(\hat{\omega} \hat{\rho}, \hat{\rho}^{1 / z}\right) .
$$

where the function $f$ encodes the presence of the higher derivative terms.

In principle, the procedure for extracting the holographic Green's function is unchanged from the prescription of (3.9). However, the higher derivative terms affect the shape of the potential, and hence may change the boundary and horizon asymptotics and possibly also introduce additional classical turning points in the bulk. In order to get a better understanding of the asymptotics, we write out the expansion

$$
\frac{1}{\hat{\rho}^{2}} f\left(\hat{\omega} \hat{\rho}, \hat{\rho}^{1 / z}\right)=\sum_{\substack{i, j \\ i+j>2}} \lambda_{i, j} \hat{\omega}^{i} \hat{\rho}^{i+j / z-2},
$$

where $i$ and $j$ count the number of temporal and spatial derivatives, respectively. The restriction $i+j>2$ ensures that $f$ only comprises the higher derivative contributions. Note that the coefficients $\lambda_{i, j}$ are dimensionless, although (after restoring units) we typically expect $\lambda_{i, j} \sim(\ell / L)^{i+j-2}$, where $\ell$ is some microscopic scale and $L$ is the curvature scale of the Lifshitz bulk, such that $\ell \ll L$.

Focusing first on the boundary at $\hat{\rho}=0$, we see that the behavior of the potential (3.10) remains dominated by the $1 / \hat{\rho}^{2}$ term, since $i+j>2$ in the derivative expansion. Thus the boundary scaling behavior remains unchanged from (3.7), and the relation of the scaling dimension to $\nu$ is unaffected by the higher order terms.

The horizon behavior, on the other hand, is considerably different. Since the horizon is located at $\hat{\rho} \rightarrow \infty$, and the expansion (3.11) in general contains positive powers of $\hat{\rho}$, the successive higher derivative terms will become more and more dominant at the horizon. Furthermore, the potential will generically go to $\pm \infty$ at the horizon, depending on the sign of $\lambda_{i, j}$ of the dominant term. As a result, strictly speaking, the perturbative expansion of the scalar equation breaks down near the horizon. Nevertheless, we now argue that the 
holographic Green's function can be extracted from the solution of the higher derivative equation in a controlled manner.

\subsection{Consistency of the higher derivative expansion}

At the two-derivative level, the Schrödinger potential (3.6) is monotonically decreasing as we move into the interior of the bulk, and there is a single classical turning point located at $\hat{\rho}_{0}$ where $\hat{U}_{0}\left(\rho_{0}\right)=0$. For $\hat{\rho}<\hat{\rho}_{0}$, the solution connects to the power-law behavior (3.7) at the boundary, while for $\hat{\rho}>\hat{\rho}_{0}$, the solution is oscillatory, and infalling boundary conditions are chosen at the horizon.

Ignoring the shift of $\nu^{2}$ in (3.6), there are two competing power laws in $\hat{U}$, namely $\nu^{2} / \hat{\rho}^{2}$ and $1 / \hat{\rho}^{2-2 / z}$, and the behavior of the solution depends on which of the power laws dominates at the classical turning point. We define the crossover point as $\hat{\rho}_{*}=\nu^{z}$, which is the location where the two terms become comparable. There are two distinct cases to consider:

1. For $\hat{\omega} \gg \nu^{1-z}$, the classical turning point is located at $\hat{\rho}_{0} \approx \nu / \hat{\omega} \ll \hat{\rho}_{*}$. This point is close to the boundary, and the $1 / \hat{\rho}^{2}$ potential ensures a power law behavior without exponential suppression. The holographic Green's function is "featureless", and behaves as $\mathcal{G} \sim \hat{\omega}^{2 \nu}$.

2. For $\hat{\omega} \ll \nu^{1-z}$, the classical turning point is instead located at $\hat{\rho}_{0} \approx \omega^{-z /(z-1)} \gg \hat{\rho}_{*}$. The Green's function now probes deep into the bulk, and can have non-trivial features. Note that the wavefunction has exponential behavior in the region $\hat{\rho}_{*}<\hat{\rho}<\hat{\rho}_{0}$, leading to an effective decoupling of the boundary from the horizon [5].

We now consider the effect of the higher derivative terms, encoded in the function $f$ in (3.11). Although this function dominates at the horizon, we nevertheless consider a formal perturbative expansion of the Schrödinger problem in the couplings $\lambda_{i, j}$. Of course, the higher order terms will dominate the wavefunction near the horizon. However, it is important to realize that the holographic Green's function is not determined by the wavefunction at the horizon, but by its asymptotic behavior at the boundary. Infalling boundary conditions are needed at the horizon, but this can be imposed consistently at each order in the perturbative expansion. These infalling conditions will be seen in the boundary Green's function, but will not dominate over lower orders in the expansion.

Although a formal perturbative expansion can be used to solve the bulk scalar equation, the expansion of $\mathcal{G}$ in the couplings $\lambda_{i, j}$ will only be sensible if the corrections can be kept small. Obviously this cannot be true globally, as the higher derivative terms typically dominate near the horizon. However, as one can see for example by using the WKB approximation (see section 4 and [5]), the holographic Green's function only gives us information about physics between the boundary and the classical turning point $\hat{\rho}_{0}$, where the wavefunction changes from exponential to oscillating behavior. Hence all that is necessary is to ensure that $f$ remains small compared to the leading order potential $\hat{U}_{0}$ only for $\hat{\rho} \leq \hat{\rho}_{0}$. The specifics of this condition depend on whether we are in the high or low frequency regime. We consider these two cases separately: 
1. In the high frequency regime $\left(\hat{\omega} \gg \nu^{1-z}\right)$, the dominant term in $\hat{U}_{0}$ is $\nu^{2} / \hat{\rho}^{2}$. Since this term is decaying, while at the same time $f$ becomes more important as we move away from the boundary, we only need to demand that $f$ is small compared to $\nu^{2} / \hat{\rho}^{2}$ at the classical turning point. This gives rise to the condition $f\left(\hat{\omega} \hat{\rho}_{0}, \hat{\rho}_{0}^{1 / z}\right) \ll \nu^{2}$, which may be satisfied by taking $(\ell / L) \nu \ll 1$, where we have assumed the expansion (3.11) along with the behavior of the couplings $\lambda_{i, j} \sim(\ell / L)^{i+j-2}$. As we may see from (3.4), the scale of $\nu$ is set by $m L$. Therefore, the condition for a valid expansion is equivalent to demanding $m \ell \ll 1$. We conclude that in this case, higher derivative corrections are under perturbative control provided the bulk couplings satisfy $m \ell \ll 1$. This behavior is very much like the relativistic $z=1$ case, since in both situations the $\nu^{2} / \hat{\rho}^{2}$ potential dominates up to the classical turning point.

2. In the low frequency regime $\left(\hat{\omega} \ll \nu^{1-z}\right)$, we need to compare $f$ with the $1 / \hat{\rho}^{2-2 / z}$ term in $\hat{U}_{0}$. Once again, we only need to consider the magnitude of $f$ at the classical turning point. The condition is now $f\left(\hat{\omega} \hat{\rho}_{0}, \hat{\rho}_{0}^{1 / z}\right) \ll \hat{\rho}_{0}^{2 / z}$, which gives rise to the requirement

$$
\hat{\omega} \gg\left(\frac{\ell}{L}\right)^{z-1}
$$

As $\hat{\omega}$ is taken smaller and smaller, we need to take higher and higher order corrections into account. As a result, the perturbative expansion breaks down at small $\hat{\omega}$, and results computed in this regime will not be robust against higher derivative corrections. Physically, what happens is that as $\hat{\omega} \rightarrow 0$, we probe closer and closer to the horizon, and it is precisely there where the higher derivative corrections dominate.

Hence, as long as the scale of the bulk higher derivative corrections satisfies $m \ell \ll 1$, the perturbative expansion of the boundary Green's function makes sense for dimensionless frequencies $\hat{\omega} \gg(\ell / L)^{z-1}$. For lower frequencies, the higher derivative terms start dominating.

This feature of higher derivative terms becoming more pronounced at the horizon is not restricted to the Lifshitz background, but is in fact fairly general and shows up in, e.g., the pure AdS and Schwarzschild-AdS cases. While the pure AdS case tends to be robust against higher derivatives because of conformal invariance, more care may be needed in the case of holography at non-zero temperature [14-21]. Transport coefficients, such as the shear viscosity, may be extracted using the Kubo formula, which is evaluated at $|\vec{k}|=0$ before sending $\omega \rightarrow 0$. Since this is consistent with (3.12), the perturbative expansion for transport coefficients is valid. At the same time, however, more care may be needed when analyzing general hydrodynamic modes, which are defined for both $\omega$ and $\vec{k}$ small, but nonzero (see e.g. [22]).

\section{WKB analysis of the spectral function}

In this section, we study the holographic spectral function of a probe scalar in Lifshitz spacetime, in the presence of higher derivative corrections. To determine the effect of 
higher derivatives on the retarded Green's function, we consider a probe scalar with an effective potential of the form

$$
\hat{U}=\frac{\nu^{2}-1 / 4}{\hat{\rho}^{2}}+\frac{1}{\hat{\rho}^{2-2 / z}}-\hat{\omega}^{2}+\sum_{i+j>2} \lambda_{i, j} \hat{\omega}^{i} \hat{\rho}^{i+j / z-2} .
$$

The last term encodes an infinite set of higher derivative corrections to the equation of motion, where the $(i, j)$ term corresponds to $i$ temporal and $j$ spatial derivatives. The size of the coefficients is expected to be set by a microscopic length scale $\ell$, so that (after restoring units of $L) \lambda_{i, j} \sim(\ell / L)^{i+j-2}$. Since it is in general not possible to solve the corresponding Schrödinger equation for the potential (4.1) analytically, we will make use of the WKB approximation to obtain an approximate solution. This method can be used to calculate the imaginary part of the retarded Green's function, which is proportional to the spectral function. After switching to the $\hat{\rho}$ coordinates defined in (3.5), the spectral function can be approximated by ${ }^{1}[5]$

$$
K^{-1} \operatorname{Im} G_{R}(\omega, \vec{k}) \approx|\vec{k}|^{2 \nu z} \lim _{\epsilon \rightarrow 0} \epsilon^{-2 \nu} e^{-2 S} .
$$

Here $K$ is a normalization constant and

$$
S=\int_{\epsilon}^{\hat{\rho}_{0}} d \hat{\rho} \sqrt{\hat{U}(\hat{\rho})+\frac{1}{4 \hat{\rho}^{2}}} .
$$

The additional $1 / \hat{\rho}^{2}$ term is equivalent to an effective shift $\nu^{2} \rightarrow \nu^{2}+\frac{1}{4}$, which is necessary for consistency of the WKB approximation for $1 / x^{2}$ potentials [7]. The integral is taken from a UV cutoff $\epsilon$ to the classical turning point $\hat{\rho}_{0}$. The WKB approximation for the imaginary part of the rescaled Green's function defined in (2.2) is given by

$$
K^{-1} \operatorname{Im} \mathcal{G}(\hat{\omega}) \approx \lim _{\epsilon \rightarrow 0} \epsilon^{-2 \nu} e^{-2 S} .
$$

This expression is valid for a potential with only one classical turning point, such that the wavefunction is oscillating near the horizon and tunnels towards the boundary. Close to the boundary, the $1 / \hat{\rho}^{2}$ part of the potential leads to a power-law scaling of the wavefunction, which is stripped off by the factor of $\epsilon^{-2 \nu}$ in (4.4). We can use (4.4) to determine the imprint of higher derivative corrections on the spectral function, provided that $\lambda_{i, j}<0$. In this case, the potential goes to $-\infty$ at the horizon, but the wavefunction still remains oscillating and we can consistently impose infalling boundary conditions. Later we will argue that (4.4) can in fact be used to provide a formal expansion for corrections with arbitrary sign.

In order to perform a perturbative expansion of the WKB integral (4.3) in terms of $\lambda_{i, j}$, we need the higher derivative corrections to be subdominant compared to the other terms in $\hat{U}$, at least in the domain of integration. We therefore demand

$$
\lambda_{i, j} \hat{\omega}^{i} \hat{\rho}^{i+j / z} \ll \nu^{2}, \quad \lambda_{i, j} \hat{\omega}^{i} \hat{\rho}^{i+j / z} \ll \hat{\rho}^{2 / z}
$$

\footnotetext{
${ }^{1}$ The additional prefactor of $|\vec{k}|^{2 \nu z}$ arises from letting $\epsilon \rightarrow|\vec{k}|^{-z} \epsilon$, which is the proper UV cutoff needed to cancel the log-divergence of the integral.
} 
for all $0<\hat{\rho} \leq \hat{\rho}_{0}$ (see also the discussion in section 3.2). We can already see that this imposes an $\hat{\omega}$-dependent condition on the coefficients $\lambda_{i, j}$, which we will make more explicit in what follows.

We can now determine the leading order correction to $\operatorname{Im} \mathcal{G}(\hat{\omega})$ by formally expanding the WKB integral in terms of the $\lambda_{i, j}$. At leading order, the higher derivative contributions are linear, so for our purposes it will be enough to drop the sum in (4.1) and only consider the effect of a single correction term with fixed $(i, j)$. In a realistic model with a tower of higher derivative corrections, one may obtain a perturbative expansion for $\operatorname{Im} \mathcal{G}(\hat{\omega})$ by summing up the individual contributions, keeping in mind that if there are corrections at different order (e.g. $\alpha^{\prime}$ and $\left(\alpha^{\prime}\right)^{2}$ ), one may have to go beyond linear order to study the effect of all correction terms.

A consistent expansion in $\lambda_{i, j}$ requires expanding both the integrand and the upper bound $\hat{\rho}_{0}$, since the location of the turning point depends on the details of the correction terms. Writing $S=S^{(0)}+\delta S$, where $S^{(0)}$ is the two-derivative integral with $\lambda_{i, j}=0$, we find (see appendix A for a rigorous derivation):

$$
\begin{aligned}
S^{(0)} & =\int_{\epsilon}^{\hat{\rho}_{0}^{(0)}} d \hat{\rho} \sqrt{\frac{\nu^{2}}{\hat{\rho}^{2}}+\frac{1}{\hat{\rho}^{2-2 / z}}-\hat{\omega}^{2}}, \\
\delta S & \approx \int_{\epsilon}^{\hat{\rho}_{0}^{(0)}} d \hat{\rho} \frac{\lambda_{i, j} \hat{\omega}^{i} \hat{\rho}^{i+j / z-2}}{2 \sqrt{\frac{\nu^{2}}{\hat{\rho}^{2}}+\frac{1}{\hat{\rho}^{2-2 / z}}-\hat{\omega}^{2}}}
\end{aligned}
$$

where $\hat{\rho}_{0}^{(0)}$ is the turning point for the case $\lambda_{i, j}=0$, i.e. the solution of

$$
\frac{\nu^{2}}{\hat{\rho}_{0}^{2}}+\frac{1}{\hat{\rho}_{0}^{2-2 / z}}-\hat{\omega}^{2}=0,
$$

and we expanded up to linear order in $\lambda_{i, j}$. The large and small $\hat{\omega}$-behavior of the unperturbed integral $S^{(0)}$ was computed in [7]:

$$
\begin{aligned}
& S^{(0)}\left(\hat{\omega} \gg \nu^{1-z}\right) \approx-\nu-\nu \log \left(\frac{\epsilon}{2 \nu}\right) \\
& S^{(0)}\left(\hat{\omega} \ll \nu^{1-z}\right) \approx-z \nu+z \nu \log (2 \nu)+\nu(z-1) \log z+\frac{\sqrt{\pi} \Gamma\left(\frac{1}{2(z-1)}\right)}{z \Gamma\left(\frac{z}{2(z-1)}\right)} \hat{\omega}^{-\frac{1}{z-1}} .
\end{aligned}
$$

Let us now calculate the leading correction (4.7) in the same limits. For $\hat{\omega} \gg \nu^{1-z}$, the unperturbed turning point lies at $\hat{\rho}_{0}^{(0)} \approx \nu / \hat{\omega}$, which is well within the region where the $1 / \hat{\rho}^{2}$ term dominates over $1 / \hat{\rho}^{2-2 / z}$. Hence we can approximate the integral as

$$
\delta S \approx \int_{\epsilon}^{\nu / \hat{\omega}} d \hat{\rho} \frac{\lambda_{i, j} \hat{\omega}^{i} \hat{\rho}^{i+j / z-2}}{2 \sqrt{\frac{\nu^{2}}{\hat{\rho}^{2}}-\hat{\omega}^{2}}} .
$$

Letting $x \equiv \hat{\omega} \hat{\rho} / \nu$, we find

$$
\delta S \approx \nu \lambda_{i, j} \nu^{i+j-2}\left(\frac{\nu^{1-z}}{\hat{\omega}}\right)^{\frac{j}{z}} \int_{\frac{\epsilon \hat{\omega}}{\nu}}^{1} d x \frac{x^{i+\frac{j}{z}-2}}{2 \sqrt{\frac{1}{x^{2}}-1}} .
$$


For $\hat{\omega} \gg \nu^{1-z}$, correction terms with $j \neq 0$ are highly suppressed. After taking the UV cutoff $\epsilon$ to zero, we therefore have

$$
\delta S \approx \delta_{j, 0} c_{i} \lambda_{i, 0} \nu^{i-1}+O\left(\frac{\nu^{1 / z-1}}{\hat{\omega}^{1 / z}}\right),
$$

where

$$
c_{i}=\int_{0}^{1} d x \frac{x^{i-1}}{2 \sqrt{1-x^{2}}}=\frac{\sqrt{\pi} \Gamma\left(\frac{i}{2}\right)}{4 \Gamma\left(\frac{i+1}{2}\right)} .
$$

Using (4.13) and the unperturbed result (4.9), we arrive at the final answer

$$
K^{-1} \operatorname{Im} \mathcal{G}\left(\hat{\omega} \gg \nu^{1-z}\right) \approx C \hat{\omega}^{2 \nu}, \quad C=(2 \nu)^{-2 \nu} \exp \left[2 \nu\left(1-\delta_{j, 0} c_{i} \lambda_{i, 0} \nu^{i-2}+\cdots\right)\right]
$$

where the ellipsis indicates terms that are higher order in $\lambda$. The scaling of $\mathcal{G}$ with $\hat{\omega}^{2 \nu}$ reflects the fact that at large frequencies, the Green's function $G_{R}(\omega, \vec{k})=|\vec{k}|^{2 \nu z} \mathcal{G}(\hat{\omega})$ becomes independent of $\vec{k}$ (see the discussion in section 2). The higher derivatives simply renormalize the numerical prefactor in a controlled way. The size of the higher derivative corrections at large $\hat{\omega}$ is controlled by

$$
\lambda_{i, 0} \nu^{i-2} \sim\left(\frac{\ell}{L} \nu\right)^{i-2} \sim(m \ell)^{i-2}
$$

where $i>2$ is the number of temporal derivatives. Note that $\lambda_{i, 0} \nu^{i-2} \ll 1$ is precisely what is required for the higher derivative corrections to be small up to the classical turning point $\hat{\rho}_{0}$, in the limit of large $\hat{\omega}$, as one can see by evaluating (4.5) at $\hat{\rho}_{0}$ in this limit, and noting that the unperturbed potential is monotonically decreasing.

We now turn to calculating the higher derivative corrections in the case of small frequencies $\left(\hat{\omega} \ll \nu^{1-z}\right)$. In this case, the unperturbed classical turning point lies at $\hat{\rho}_{0}^{(0)} \approx \hat{\omega}^{-z /(z-1)}$. We can split up the integral (4.7) in the following way [7]: Let $\hat{\rho}_{*}=\nu^{z}$ be the crossover scale, defined in the beginning of section 3.2 , at which the two different terms in the potential (3.10), $\nu^{2} / \hat{\rho}^{2}$ and $1 / \hat{\rho}^{2-2 / z}$, become comparable. Since $\hat{\omega} \ll \nu^{1-z}$, we can then introduce a regulator scale $\hat{\rho}_{r}$ such that $\hat{\rho}_{*} \ll \hat{\rho}_{r} \ll \hat{\rho}_{0}^{(0)}$, and split up the WKB integral in (4.7) as

$$
\int_{\epsilon}^{\hat{\rho}_{0}^{(0)}}=\int_{\epsilon}^{\hat{\rho}_{r}}+\int_{\hat{\rho}_{r}}^{\hat{\rho}_{0}^{(0)}} .
$$

The first of the integrals above is taken over $\epsilon \leq \hat{\rho} \leq \hat{\rho}_{r} \ll \hat{\rho}_{0}^{(0)}$, so we can approximate the potential in this region as

$$
\hat{U} \approx \frac{\nu^{2}}{\hat{\rho}^{2}}+\frac{1}{\hat{\rho}^{2-2 / z}}+\lambda_{i, j} \hat{\omega}^{i} \hat{\rho}^{i+j / z-2} .
$$

On the other hand, the second integral is taken over $\hat{\rho}_{r} \leq \hat{\rho} \leq \hat{\rho}_{0}^{(0)}$, so in this region we can write

$$
\hat{U} \approx \frac{1}{\hat{\rho}^{2-2 / z}}-\hat{\omega}^{2}+\lambda_{i, j} \hat{\omega}^{i} \hat{\rho}^{i+j / z-2} .
$$


Using these approximations, we find

$$
\begin{aligned}
\delta S & =\delta S_{1}+\delta S_{2}, \\
& \approx \int_{\epsilon}^{\hat{\rho}_{r}} d \hat{\rho} \frac{\lambda_{i, j} \hat{\omega}^{i} \hat{\rho}^{i+j / z-2}}{2 \sqrt{\frac{\nu^{2}}{\hat{\rho}^{2}}+\frac{1}{\hat{\rho}^{2-2 / z}}}}+\int_{\hat{\rho}_{r}}^{\hat{\rho}_{0}^{(0)}} d \hat{\rho} \frac{\lambda_{i, j} \hat{\omega}^{i} \hat{\rho}^{i+j / z-2}}{2 \sqrt{\frac{1}{\hat{\rho}^{2-2 / z}}-\hat{\omega}^{2}}} .
\end{aligned}
$$

Letting $u=\frac{1}{\nu^{2}} \hat{\rho}^{2 / z}$, the first integral can be written as

$$
\delta S_{1}=\frac{z \nu}{4} \lambda_{i, j} \nu^{i+j-2}\left(\frac{\hat{\omega}}{\nu^{1-z}}\right)^{i} \int_{u_{\epsilon}}^{u_{r}} d u \frac{u^{\frac{z}{2} i+\frac{j}{2}-1}}{\sqrt{1+u}}
$$

where the integration bounds are $u_{\epsilon}=\epsilon^{2 / z} / \nu^{2} \rightarrow 0$ and $u_{r}=\left(\hat{\rho}_{r} / \hat{\rho}_{*}\right)^{2 / z} \gg 1$. In the small frequency limit $\hat{\omega} \ll \nu^{1-z}$, the correction term is highly suppressed unless $i=0$. Hence we have

$$
\delta S_{1} \approx \delta_{i, 0} \frac{z \nu}{4} \lambda_{0, j} \nu^{j-2} \int_{u_{\epsilon}}^{u_{r}} d u \frac{u^{\frac{j}{2}-1}}{\sqrt{1+u}}+O\left(\frac{\hat{\omega}}{\nu^{1-z}}\right) .
$$

The remaining integral is divergent as $u_{r} \rightarrow \infty$. However, one can show that the contribution of the upper bound cancels with that from the lower bound of $\delta S_{2}$, since $u_{r}$ is after all a fictitious regulator scale. Hence the only contribution of (4.22) to $\delta S$ is due to evaluating the integral at the lower bound $u_{\epsilon} \rightarrow 0$ :

$$
\delta S_{1} \rightarrow-\delta_{i, 0} \frac{z \nu}{4} d_{j} \lambda_{0, j} \nu^{j-2}+O\left(\frac{\hat{\omega}}{\nu^{1-z}}\right)
$$

where

$$
d_{j}=\int^{u=0} d u \frac{u^{\frac{j}{2}-1}}{\sqrt{1+u}} .
$$

This contribution is finite; in particular there are no $\log \epsilon$ terms, which would affect the boundary scaling. Similar to the large $\hat{\omega}$ case, higher derivative corrections are controlled by terms of order $\sim \lambda \nu^{n-2}$, where $n$ counts the number of derivatives. This becomes qualitatively different when considering $\delta S_{2}$, which captures the contribution of higher derivative corrections deep in the bulk. Letting $x=\hat{\omega}^{z /(z-1)} \hat{\rho}$, we obtain

$$
\delta S_{2}=\frac{1}{2} \hat{\omega}^{-\frac{1}{z-1}} e_{i, j} \lambda_{i, j} \hat{\omega}^{-\frac{1}{z-1}(i+j-2)},
$$

where

$$
e_{i, j}=\int_{\hat{\rho}_{r} / \hat{\rho}_{0}^{(0)}}^{1} d x \frac{x^{i-1+\frac{j-1}{z}}}{\sqrt{1-x^{2-\frac{2}{z}}}} .
$$

When expanding the lower bound in powers of $\hat{\rho}_{r} / \hat{\rho}_{0}^{(0)}$, each term is designed to cancel with the corresponding contribution from $\delta S_{1}$. Instead of carrying out this cancellation explicitly, we can therefore let

$$
e_{i, j} \rightarrow \int_{0}^{1} d x \frac{x^{i-1+\frac{j-1}{z}}}{\sqrt{1-x^{2-\frac{2}{z}}}}=\frac{\sqrt{\pi} \Gamma\left(\frac{i z+j-1}{2(z-1)}\right)}{\left(2-\frac{2}{z}\right) \Gamma\left(\frac{(i+1) z+j-2}{2(z-1)}\right)},
$$


together with the prescription (4.23). Using (4.23), (4.25) and the zeroth order result (4.10), we arrive at the final answer

$$
K^{-1} \operatorname{Im} \mathcal{G}\left(\hat{\omega} \ll \nu^{1-z}\right) \approx D \exp \left[-\hat{\omega}^{-\frac{1}{z-1}} E(\hat{\omega})\right],
$$

where

$$
\begin{aligned}
D & =(2 \nu)^{-2 z \nu} z^{2 \nu(1-z)} \exp \left[2 z \nu\left(1+\delta_{i, 0} d_{j} \lambda_{0, j} \nu^{j-2}+\cdots\right)\right], \\
E(\hat{\omega}) & =\frac{\sqrt{\pi} \Gamma\left(\frac{1}{2(z-1)}\right)}{z \Gamma\left(\frac{z}{2(z-1)}\right)}+e_{i, j} \lambda_{i, j} \hat{\omega}^{-\frac{1}{z-1}(i+j-2)}+\cdots
\end{aligned}
$$

Here the ellipses indicate terms that are higher order in $\lambda$. We see that the higher derivative terms have two distinct effects: First, corrections with $i=0$, which correspond to purely spatial derivatives, affect the overall normalization $D$ of the spectral function. Second, and more importantly, higher derivative corrections with any $i$ and $j$ change the behavior of the spectral function as $\hat{\omega} \rightarrow 0$, encoded in $E(\hat{\omega})$. The $\hat{\omega}$-dependent correction terms become more and more important at small frequencies, and eventually the perturbative expansion breaks down. This was to be expected, since at small $\hat{\omega}$, the spectral function probes deep into the bulk, where higher derivatives dominate. However, recall that the coupling constants $\lambda_{i, j}$ are generically given by a ratio of a microscopic versus macroscopic length scale, $\lambda_{i, j} \sim(\ell / L)^{i+j-2}$. It is thus possible to keep the corrections in (4.28) small by demanding

$$
\nu^{1-z} \gg \hat{\omega} \gg\left(\frac{\ell}{L}\right)^{z-1}
$$

This is precisely the bound we argued for in section 3.2. Since the condition (4.5), evaluated at large $\hat{\omega}$, also guarantees that $\ell \nu / L \ll 1$ (see the discussion around (4.16) and section 3.2), there is a wide range of frequencies that satisfy the inequality (4.30). For frequencies within this range, (4.28) is a universal result: The spectral function behaves as $\sim \exp \left(-\right.$ const. $\left.\cdot \hat{\omega}^{-1 /(z-1)}\right)$, and there are both constant and $\hat{\omega}$-dependent corrections that can be computed order by order in perturbation theory. The naive limit $\hat{\omega} \rightarrow 0$ is non-universal, since higher derivative corrections cannot be kept under control.

The procedure for calculating higher derivative corrections to the spectral function outlined in this section can in principle be applied to arbitrary corrections of the form (4.1). Note, however, that since we generally expect an infinite number of such corrections, going beyond leading order in $\ell / L$ may require expanding (4.3) to the appropriate order.

Finally, let us comment on the sign of $\lambda_{i, j}$. In the analysis above, we assumed that $\lambda_{i, j}<0$, so that the wavefunction is always oscillating at the horizon, and no additional turning points are introduced. If $\lambda_{i, j}$ is positive, the wavefunction is tunneling in the deep IR, leading to another tunneling contribution $S_{\mathrm{IR}}$ to the spectral function (4.2). At large enough $\hat{\rho}$, the higher derivative corrections will always dominate the potential, so $S_{\text {IR }}$ does not have a perturbative expansion in $\lambda_{i, j}$. This is simply a consequence of the fact that the potential in the IR is always sensitive to all of the (in principle infinitely 
many) coefficients that appear in the series of higher derivative corrections, and we cannot solve the equation of motion perturbatively in the IR. It therefore seems that one cannot trust our analysis in the case of generic corrections with arbitrary sign. However, one can circumvent this problem in the following way: For any given $\hat{\omega}$, we can define a regulator surface at $\hat{\rho}=\hat{\rho}_{h}(\hat{\omega}) \gg \hat{\rho}_{0}$, such that higher derivative corrections are still small at $\hat{\rho}_{h}$, i.e.

$$
\lambda_{i, j} \hat{\omega}^{i} \hat{\rho}_{h}^{i+j / z} \ll \nu^{2}, \quad \lambda_{i, j} \hat{\omega}^{i} \hat{\rho}_{h}^{i+j / z} \ll \hat{\rho}_{h}^{2 / z} .
$$

This guarantees that the wavefunction is still oscillating at $\hat{\rho}_{h}$, even though eventually higher derivatives may cause the potential to bend upwards again. Ignoring the (unknown) behavior of the wavefunction in the deep IR, we only impose infalling boundary conditions at $\hat{\rho}_{h}$, instead of $\hat{\rho} \rightarrow \infty$. The surface at $\hat{\rho}_{h}$ thus becomes an "effective horizon", where the wavefunction is infalling:

$$
\psi\left(\hat{\rho} \rightarrow \hat{\rho}_{h}\right) \approx a e^{i \Phi(\hat{\rho})} .
$$

Here $\Phi$ is an increasing function of $\hat{\rho}$. The retarded Green's function can then be computed using the usual formula (3.9), and the spectral function can be calculated approximately using the WKB-formula (4.2). On a practical level, this regularization prescription amounts to simply taking (4.2) for granted, and formally expanding the WKB integral $S$ in $\lambda_{i, j}$, without worrying about the dynamics close to the horizon.

\section{$5 \quad$ Field theory models with $z=2$}

As we have demonstrated, a holographic computation of the spectral function yields the universal low-frequency behavior $\chi \sim \exp \left(-\right.$ const. $\left.\cdot \hat{\omega}^{-1 /(z-1)}\right)$, provided $\hat{\omega}$ is in the range (4.30), where the higher derivative corrections are controlled. From a field theory point of view, such an exponential behavior is not expected to arise at any finite perturbative order, but can show up non-perturbatively. This, of course, fits the framework of non-relativistic holography, where the field theory dual is expected to involve strong correlations.

In this section, we explore two field theoretic models exhibiting $z=2$ Lifshitz scaling. The first model is the quadratic band crossing model of [12], and the second is the quantum Lifshitz model [13]. Our strategy will be to identify phase-space regions with nonzero decay rates for bosonic quasi-particles, which, according to the optical theorem, will contribute to the imaginary part of the corresponding bosonic Green's functions, and hence the spectral function. In both models, we confirm the presence of exponential suppression in the spectral function at small $\hat{\omega}$, in agreement with the holographic computation.

\subsection{The quadratic band crossing model}

To set up the quadratic band crossing model, let us start with a massless Dirac theory in $2+1$ dimensions, with action ${ }^{2}$

$$
S=\int d \vec{x} d t\left[\bar{\Psi}\left(i \gamma_{0} \partial_{0}-i \gamma_{1} \partial_{x}-i \gamma_{2} \partial_{y}\right) \Psi-g \psi_{1}^{\dagger} \psi_{2}^{\dagger} \psi_{2} \psi_{1}\right]
$$

\footnotetext{
${ }^{2}$ Note that we use signature $(+,-,-)$ for the field theory.
} 
Here $\Psi=\left(\psi_{1}, \psi_{2}\right)^{T}$ is a two-component spinor and $\bar{\Psi}=\Psi^{\dagger} \gamma_{0}$. The $2+1$ dimensional Dirac matrices are given by

$$
\gamma_{0}=\left(\begin{array}{cc}
0 & -i \\
i & 0
\end{array}\right), \quad \gamma_{1}=\left(\begin{array}{cc}
0 & i \\
i & 0
\end{array}\right), \quad \gamma_{2}=\left(\begin{array}{cc}
-i & 0 \\
0 & i
\end{array}\right) .
$$

The interaction term in (5.1) is the only four-fermi term allowed for a two-component spinor. In the IR, this $\psi^{4}$ term is also the most relevant interaction term in the RG sense. At the Gaussian fixed point, this theory is conformally invariant with dynamical critical exponent $z=1$. By setting the speed of light to unity, the theory contains only one control parameter, which is the interaction strength $g$.

The quadratic band crossing model generalizes the above to form a scaling invariant model with $z=2$ [12]. It does so by replacing the derivatives in the Dirac theory (5.1) by the following operators:

$$
\begin{aligned}
& i \partial_{0} \rightarrow i \partial_{0}+t_{0} \nabla^{2}, \\
& i \partial_{x} \rightarrow-t_{1}\left(\partial_{x}^{2}-\partial_{y}^{2}\right), \\
& i \partial_{y} \rightarrow-2 t_{2} \partial_{x} \partial_{y},
\end{aligned}
$$

where $\nabla^{2}=\partial_{x}^{2}+\partial_{y}^{2}$ and $t_{0}, t_{1}$ and $t_{2}$ are real parameters. After this substitution, we obtain a model with $z=2$ :

$$
S=\int d \vec{x} d t\left\{\bar{\Psi}\left[\gamma_{0}\left(i \partial_{0}+t_{0} \nabla^{2}\right)+\gamma_{1} t_{1}\left(\partial_{x}^{2}-\partial_{y}^{2}\right)+2 \gamma_{2} t_{2} \partial_{x} \partial_{y}\right] \Psi-g \psi_{1}^{\dagger} \psi_{2}^{\dagger} \psi_{2} \psi_{1}\right\} .
$$

This action bears some resemblance with the original Dirac theory. However, in direct contrast to the Dirac theory, whose action only contains first-order derivatives, this model has a first order time derivative and second order spatial derivatives. As a result, space and time have different scaling dimensions, and it is straightforward to show that dimensionally $[t]=2[\vec{x}]$, corresponding to $z=2$ at the Gaussian fixed point. In condensed matter systems, this model describes band touching points with quadratic dispersions, which have been observed in bilayer graphene (see for example the review articles [23-25]); a realization has been proposed in optical lattice systems, using high angular momentum orbitals [26].

Generically, the action (5.4) contains four control parameters: $t_{0}, t_{1}, t_{2}$, and the interaction strength $g$. However, we can set one of the three $t_{i}$ 's to unity (say $t_{2}=1$ ) by rescaling. As shown in [12] and detailed in appendix $\mathrm{B}$, if we require $\mathrm{SO}(2)$ spatial rotational symmetry, then $t_{1}$ and $t_{2}$ must coincide. Furthermore, if a fermion particle-hole symmetry (i.e. charge conjugation) is enforced, then $t_{0}$ must vanish. Here we will focus on the case with $t_{0}=0$ and $t_{1}=t_{2}=1$, which preserves both the spatial rotational and charge-conjugation symmetries. In this case, the action reduces to

$$
S=\int d \vec{x} d t\left\{\bar{\Psi}\left[\gamma_{0} i \partial_{0}+\gamma_{1}\left(\partial_{x}^{2}-\partial_{y}^{2}\right)+2 \gamma_{2} \partial_{x} \partial_{y}\right] \Psi-g \psi_{1}^{\dagger} \psi_{2}^{\dagger} \psi_{2} \psi_{1}\right\}
$$

and as shown in appendix B, the free dispersion relation for the two bands is given simply by

$$
\epsilon_{ \pm}(\vec{k})= \pm k^{2}
$$


It is worth emphasizing that most of our conclusions remain valid as long as $\left|t_{0}\right|<\left|t_{1}\right|$ and $\left|t_{0}\right|<\left|t_{2}\right|$. As discussed in appendix $\mathrm{B}$, these inequalities ensure that the model has both particles and holes in the weak coupling limit (small $g$ ).

\subsubsection{Renormalization group analysis}

At tree level, the $\psi^{4}$ term in the quadratic band crossing model is irrelevant (relevant) in the IR for systems above (below) $2+1$ dimensions. In $2+1$ dimensions, $g$ is marginal at the tree level. A one-loop RG analysis indicates that a repulsive interaction $(g>0)$ is marginally relevant at IR, while an attractive interaction $g<0$ is marginally irrelevant in the IR [12]. In the Dirac theory (5.1) on the other hand, the $\psi^{4}$ term is irrelevant (relevant) in the IR for systems above (below) $1+1$ dimensions. In $1+1$ dimensions, due to the special properties of the $1+1$ conformal group, the $\psi^{4}$ term remains exactly marginal, before the system hits a Kosterlitz-Thouless transition.

\subsubsection{Boson correlation functions}

Although the model discussed above describes fermionic fields, bosonic modes can be constructed from these fermionic degrees of freedom in the form of fermion bilinears. In the particle-hole channel, we can build four different fermion bilinears (boson modes)

$$
b_{i}=\bar{\Psi} \gamma_{i} \Psi
$$

with $i=0,1,2$, and 3 , and the fourth gamma matrix is given by $\gamma_{3}=i \gamma_{0} \gamma_{1} \gamma_{2}$. Here $b_{0}$ is the fermion density operator and the other three bosonic operators can be used as order parameters for various symmetry breaking phases (nematic or quantum anomalous Hall) [12]. At the Gaussian fixed point, these bosonic modes have $z=2$, which is inherited from the fermions.

Additional bosonic modes can also be created in the particle-particle channel (e.g. $\left.\psi_{1}^{\dagger} \psi_{2}^{\dagger}\right)$, which are the order parameters for various superconducting states. In this section, we will only consider fermion bilinears in the particle-hole channel. These bosons can decay into particle-hole pairs and are thus expected to have a finite lifetime. Via the optical theorem, the existence of such decay channels is equivalent to a non-zero imaginary part of the two-point function (and thus the spectral function), generated by self-energy diagrams such as those shown in figure 1.

Although it is challenging to analytically compute these diagrams, it is straightforward to prove that for a boson with momentum $\vec{k}$, the imaginary part of each self-energy diagram can only arise when the energy $\omega$ of the boson is larger than a certain threshold. For each diagram, this threshold can be determined using energy-momentum conservation.

For example, the one loop diagram shown in figure 1(a) (the leading order correction) computes the scattering rate for a boson mode with energy $\omega$ and momentum $\vec{k}$ to decay into one particle with energy $\omega_{p}$ and momentum $\vec{k}_{p}$ and one hole with energy $\omega_{h}$ and momentum $\vec{k}_{h}$. Such a decay process can only take place when both the energy and 


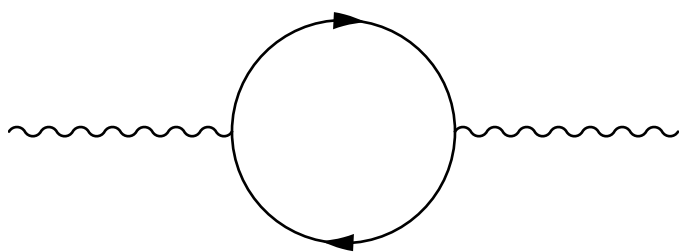

(a) A one-loop contribution to the spectral function, corresponding to the decay of a boson into one particle-hole pair.

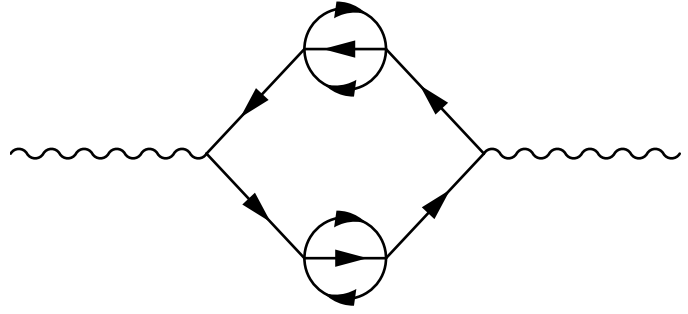

(b) A five-loop contribution to the spectral function, corresponding to the decay into three particles and three holes.

Figure 1. Self-energy corrections for the boson modes. Here, solid lines represent fermionic propagators and wiggly lines are boson propagators.

momentum conservation laws are satisfied:

$$
\begin{aligned}
\vec{k} & =\vec{k}_{p}-\vec{k}_{h}, \\
\omega & =\omega_{p}-\omega_{h}=k_{p}^{2}+k_{h}^{2} \geq \frac{k^{2}}{2} .
\end{aligned}
$$

Here we used the quadratic dispersion relation (5.6). For fixed $\vec{k}$, the momentum conservation law enforces a relation between the momentum of the particle $\vec{k}_{p}$ and that of the hole $\vec{k}_{h}$, i.e. $\vec{k}_{p}=\vec{k}+\vec{k}_{h}$. With this constraint, the energy of the particle-hole excitation $k_{p}^{2}+k_{h}^{2}$ has a lower bound of $k^{2} / 2$ (which is reached when $\vec{k}_{p}=-\vec{k}_{h}=\vec{k} / 2$ ). In other words, the energy conservation law can only be satisfied when $\omega \geq k^{2} / 2$. As a result, for $\omega \geq k^{2} / 2$, the boson can decay into a particle-hole pair, and thus have a finite lifetime, while for $\omega<k^{2} / 2$, decay is kinematically forbidden. Thus, at the one-loop level, $O\left(g^{0}\right)$, the imaginary part of the bosonic correlation function only arises for $\omega \geq k^{2} / 2$. This energy range is known as the particle-hole continuum.

When the $\psi^{4}$-interaction term is taken into consideration, the bosonic modes can decay through higher order processes (one example is shown in figure 1(b)). For these higher order diagrams, the same analysis can be utilized. At order $O\left(g^{2 n}\right)$, the energy and momentum conservation laws imply that

$$
\begin{aligned}
\vec{k} & =\sum_{i=1}^{n+1} \vec{k}_{p_{i}}-\sum_{i=1}^{n+1} \vec{k}_{h_{i}} \\
\omega & =\sum_{i=1}^{n+1} \omega_{p_{i}}-\sum_{i=1}^{n+1} \omega_{h_{i}}=\sum_{i=1}^{n+1}\left(k_{p_{i}}^{2}+k_{h_{i}}^{2}\right) \geq \frac{k^{2}}{2(n+1)} .
\end{aligned}
$$

Here we consider the decay of a bosonic mode into $n+1$ particles and $n+1$ holes (see figure 1 (b) for an example with $n=2$ ). For fixed $\vec{k}$, momentum conservation enforces a constraint on the momenta of the particles and holes. Given this constraint, the energy is minimized when the momenta are collinear, and the boson momentum $\vec{k}$ is equally 
distributed among the particles and holes. This results in a lower bound on the energy of $k^{2} / 2(n+1)$. Thus the decay is kinematically forbidden unless $\omega \geq k^{2} / 2(n+1)$.

This analysis demonstrates that, up to order of $O\left(g^{2 n}\right)$, the imaginary part of the boson correlation function only arises when the energy of the boson is above a threshold, $\omega \geq k^{2} / 2(n+1)$. Furthermore, this threshold goes down to zero for higher order diagrams as $\sim 1 /(n+1)$. Thus, if we sum the diagrammatic expansion to infinite order $(n \rightarrow \infty)$, we expect that the boson correlation function can pick up a nonzero imaginary part for any $\omega>0$.

Finally, we are ready to extract the asymptotic form of the imaginary part of the selfenergy correction at small $\omega$. For $\omega \ll k^{2}$, the imaginary part can only arise via high order process $O\left(g^{2 n}\right)$, where $n \sim k^{2} / 2 \omega$. Therefore, we expect the imaginary part at energy $\omega$ and momentum $\vec{k}$ to scale as $\sim g^{2 n} \sim g^{k^{2} / \omega}$. For sufficiently small $g$, this relation implies that the imaginary part of the self-energy correction decays to zero with the singular behavior $\sim e^{- \text {const. } / \hat{\omega}}$, where $\hat{\omega}=\omega / k^{2}$ is the dimensionless energy. This matches the $z=2$ low frequency behavior (4.28) obtained holographically.

\subsubsection{Dirac theory revisited and systems with higher $z$}

We can repeat the kinematical analysis used above for similar models with arbitrary $z \geq 1$. In this case, for $O\left(g^{2 n}\right)$, the energy-momentum conservation law becomes

$$
\begin{aligned}
\vec{k} & =\sum_{i=1}^{n+1} \vec{k}_{p_{i}}-\sum_{i=1}^{n+1} \vec{k}_{h_{i}} \\
\omega & =\sum_{i=1}^{n+1} \omega_{p_{i}}-\sum_{i=1}^{n+1} \omega_{h_{i}}=\sum_{i=1}^{n+1}\left(k_{p_{i}}^{z}+k_{h_{i}}^{z}\right) \geq \frac{k^{z}}{(2 n+2)^{z-1}} .
\end{aligned}
$$

For any $z>1$, the lower bound for having a nonzero imaginary part depends on $n$, and goes to zero as $n \rightarrow \infty$ (i.e. when considering higher and higher order diagrams). Similar to the discussion above, after summing over all the diagrams to infinite order, we find that at small $\omega$, the imaginary part of the self-energy scales as

$$
\operatorname{Im} \Pi \sim g^{\left(k^{z} / \omega\right)^{1 /(z-1)}} .
$$

For small $g$, this indicates that $\operatorname{Im} \Pi$ decays to zero as $e^{- \text {const. } \hat{\omega}^{-1 /(z-1)}}$, where now $\hat{\omega}=$ $\omega / k^{z}$, in agreement with the holographic result (4.28). This suggests that the exponential suppression of the spectral function is a generic property of Lifshitz models at $\omega \ll k^{z}$.

Note that for $z=1$, the Dirac theory is recovered, and the fate of the system is fundamentally different. As can be seen by substituting $z=1$ into (5.10), the energy threshold becomes independent of $n$. For any diagram, regardless of its order, the imaginary part arises only for $\omega \geq k$. After summing over all diagrams (to infinite order), the same lower bound of energy remains $(\omega \geq k)$. As a result, for $z=1$ the imaginary part of the correlation function vanishes identically in a finite region $\omega \leq k$, which is in sharp contrast to the $z>1$ case. This conclusion is consistent with a symmetry analysis, which tells us that at $z=1$, the Lorentz and conformal symmetries require the bosonic correlation 
function to be proportional to $(-\omega+|\vec{k}|)^{\alpha}$, where $\alpha$ is some scaling exponent. For noninteger $\alpha,(-\omega+|\vec{k}|)^{\alpha}$ is real for $\omega<|\vec{k}|$, while the imaginary part arises for $\omega>|\vec{k}|$. For $z>1$, however, the absence of the Lorentz and conformal symmetries allows for very different types of behavior.

In summary, we find that models with $z>1$ and $z=1$ belong to fundamentally different universality classes. The case with $z=1$ (i.e. Dirac) has been well understood with the help of conformal symmetry, which almost fully fixes the functional form of the correlation functions. However, for $z>1$, the absence of conformal symmetry allows for richer structure in the correlation function. For arbitrary $z>1$, we have presented an argument suggesting a characteristic exponential behavior $e^{- \text {const. } / \hat{\omega}^{1 /(z-1)}}$ for the imaginary part of the self-energy correction at low energy.

\subsubsection{Limitations of the analysis}

An exponential fall-off $\sim e^{- \text {const. } / \hat{\omega}^{1 /(z-1)}}$ of the spectral function all the way down to $\omega \rightarrow 0$ would correspond to an essential singularity of the two-point function at the origin. However, it is worth noting that there are two limitations of the analysis presented above. First, because we only considered the decay of bosonic modes into $n+1$ particle-hole pairs without taking into account the renormalization of the vertex function (i.e. the renormalization of the coupling constant $g$ ), the above analysis is not expected to give quantitatively accurate results in the extremely low (or high) energy limit. This is because, as discussed above, in $2+1$ dimensions, the coupling constant $g$ is marginally relevant or irrelevant (depending on the sign of $g$ ). For the IR or UV limit, the flow of $g$ cannot be ignored. However, because $g$ is only marginally relevant or irrelevant, the flow of $g$ is expected to be slow (i.e. logarithmic). Hence there may exist a range for $\omega$ (i.e. $\omega$ is small, but not too small) in which the RG flow of $g$ may be weak enough to be ignored, so that the analysis above can produce a reasonable estimate for the scaling behavior of $\operatorname{Im} \Pi$.

Second, in the context of QFT, the perturbation series in terms of Feynman diagrams is typically expected to be an asymptotic series. This means that our kinematical argument using loop diagrams only captures the behavior of the imaginary part of the self-energy correctly up to some finite order $O\left(g^{2 N}\right)$, where $N$ is large but finite. In particular, this implies that the scaling $\operatorname{Im} \Pi \sim g^{2 n} \sim g^{1 / \hat{\omega}^{1 /(z-1)}}$ is only valid for $n \leq N$ and thus for $\hat{\omega}$ above some cutoff $\hat{\omega}_{\star}(N)$.

Both of these points suggest that while the exponential suppression of the spectral function is a generic feature in a finite region where $\hat{\omega}$ is small, the behavior in the strict limit $\hat{\omega} \rightarrow 0$ is model-dependent. This is consistent with the observation in the gravity theory, where the would-be singular behavior of the two-point function may receive significant corrections at very small $\hat{\omega}$ from model-dependent higher derivative terms.

\subsection{The quantum Lifshitz model}

We now turn to the quantum Lifshitz model [13], which is a $z=2$ generalization of the Klein-Gordon theory. We start with the action

$$
S=\int d \vec{x} d t\left[\left(\partial_{0} \Phi\right)^{2}-\left(\nabla^{2} \Phi\right)^{2}-m \Phi^{2}-g \Phi^{4}\right] .
$$




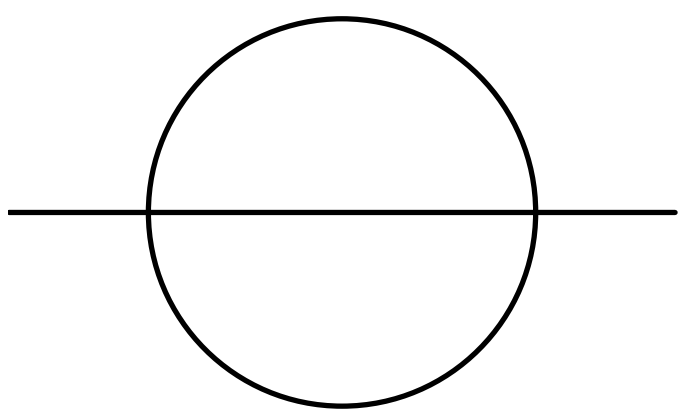

(a) The two-loop sun-set diagram contribution to the spectral function, corresponding to the decay of one boson into three bosons.

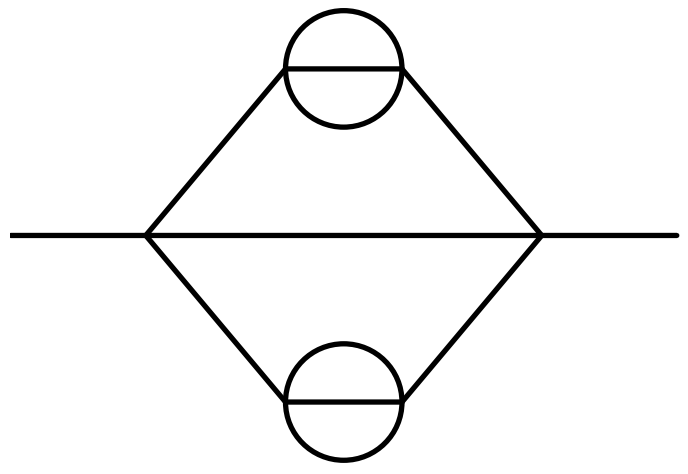

(b) A six-loop contribution to the spectral function, corresponding to the decay into seven bosons.

Figure 2. Self-energy corrections in the quantum Lifshitz model. Here, solid lines represent bosonic propagators.

Similar to the $\Phi^{4}$-model, positive (negative) $m$ corresponds to the disordered (ordered) phase respectively. The quantum Lifshitz model focusses on the quantum critical point between these two phases (at $m=0$ ), at which the system is scaling invariant. At tree level, the dimensions of the various quantities are

$$
[\omega]=2, \quad[k]=1, \quad[\Phi]=\frac{d-2}{2}, \quad[g]=6-d,
$$

where $d$ is the number of spatial dimensions. The fact that $[\omega]=2[k]$ implies $z=2$.

In the non-interacting regime $(g=0)$, the bosonic field $\Phi$ correspond to free bosons with quadratic dispersion relation

$$
\omega= \pm k^{2}
$$

and the (free) two-point correlation function is

$$
\langle\Phi(k, \omega) \Phi(-k,-\omega)\rangle=\frac{1}{\omega^{2}-k^{4}} .
$$

Similar to the previous model, we consider the decay of a bosonic mode with energy $\omega$ and momentum $\vec{k}$ into $2 n+1$ bosonic modes (with energy $\omega_{i}$ and momentum $\vec{k}_{i}$ where $i=1, \ldots, 2 n+1)$ in the $2 n$-th order diagram $O\left(g^{2 n}\right)$. Energy and momentum conservation imply

$$
\begin{aligned}
& \vec{k}=\sum_{i=1}^{2 n+1} \vec{k}_{i}, \\
& \omega=\sum_{i=1}^{2 n+1} \omega_{i}=\sum_{i=1}^{2 n+1} k_{i}^{2} \geq \frac{k^{2}}{2 n+1} .
\end{aligned}
$$


Once again, we find that the decay can only take place for $\omega$ above a threshold, which approaches zero as $n$ goes to infinity.

Using the same analysis, we see that the imaginary part of the correlation function is nonzero for any finite $\omega$, and at small $\omega$ the imaginary part is $\sim g^{2 n}$ with $n \sim k^{2} / 2 \omega$. As a result, the imaginary part scales as $\sim g^{k^{2} / \omega}$, and we again recover the non-analytic $z=2$ behavior $\sim e^{- \text {const. } / \hat{\omega}}$ as $\hat{\omega} \rightarrow 0$.

\section{Discussion}

In our previous work, we found that the spectral function for a minimally coupled scalar in a Lifshitz background was nonzero, but exponentially small, in the low-frequency regime $\omega \ll k^{z}[5]$. The analysis presented here shows that this behavior is a robust holographic prediction for field theories with Lifshitz symmetry, in the absence of further constraining symmetries. For the classes of higher derivative theories we study holographically, we generically find that the spectral function is suppressed in the low frequency region as $\chi \sim \exp \left(-\right.$ const. $\left.\cdot \hat{\omega}^{-1 /(z-1)}\right)$, so long as $\hat{\omega} \gg(\ell / L)^{z-1}$, where $\ell$ is the length scale at which higher derivatives become important.

On the field theory side, the Lifshitz scaling symmetry is a priori not expected to lead to a universal 2-point function, and perturbative calculations do not reveal any similarities either between different field theories with Lifshitz symmetry, or with the holographic theory. However, we were able to show that in both of the field theory models considered here, a simple kinematical argument involving energy-momentum conservation and a resummation of loop diagrams reveals a similar exponential suppression as predicted by holography. Furthermore, this exponential suppression is expected for any field theory containing the following three key features: The existence of particles and holes, an interaction that allows for decay channels, and a dispersion relation with $z>1$ scaling symmetry. Therefore, we expect our conclusion to be generic and applicable to a wide range of systems (with $z>1$ ) regardless of microscopic details, in agreement with the holographic prediction.

Although in both the holographic and the field theory calculation, the exponential suppression is a robust feature of the spectral function for small $\hat{\omega}$, the strict limit $\hat{\omega} \rightarrow 0$ is non-universal in both cases. In the holographic calculation, the model-dependence enters through higher derivative terms, which introduce corrections whose size can be quantified precisely (see equation (4.30)). However, the precise regime of validity of the field theory calculations is less clear. In both of the models considered here, the flow of the coupling constant $g$ can no longer be neglected when taking the exact limit $\hat{\omega} \rightarrow 0$. Instead of just being a simple exponential, the exact (nonperturbative) spectral function will therefore have a more complicated dependence on $\hat{\omega}$. Naively, one may expect a dependence of the form

$$
\operatorname{Im} \mathcal{G} \sim g(\hat{\omega})^{-1 /(z-1)} .
$$

In $2+1$ dimensions, the coupling $g$ is marginal, and we expect $g$ to depend only weakly on $\hat{\omega}$, so that the spectral function still shows an approximately exponential behavior. It would be interesting to further study the renormalization group flow of $g$ to make a precise statement about the range of $\hat{\omega}$ for which this is the case. Along the same lines, 
in order to put a precise lower bound on $\hat{\omega}$, it would be important to account for the fact that the perturbative expansion is in fact only an asymptotic series (see the discussion in section 5.1.4).

In our field theory calculation, we found that $\operatorname{Im} \mathcal{G} \sim g^{1 / \hat{\omega}^{1 /(z-1)}}$, so that exponential suppression in fact only arises for $g \ll 1$. It is important to note that this is not in contradiction to AdS/CFT being a weak-strong coupling duality. The strong coupling nature of the field theory does not necessarily mean that the parameter $g$ has to be chosen large, but rather that strong correlations (for example seen as long-range interactions) may emerge dynamically. This feature is familiar from the standard case of relativistic AdS/CFT, where it is not $g_{\mathrm{YM}}$ itself that is taken large, but rather the 't Hooft coupling $g_{\mathrm{YM}}^{2} N \gg 1$. In order to better understand the relation between strong/weak coupling on the field theory/gravity side in non-relativistic AdS/CFT, it would be desirable to develop a more precise version of the holographic dictionary for this case.

Although we have chosen not to consider higher derivatives in the radial direction $\rho$ beyond second order, this is in fact not a true limitation of the perturbative analysis. Assuming we are only interested in solutions to the higher derivative equation that are perturbatively connected to the lowest order (i.e. the two-derivative) equation, we may always eliminate higher derivatives by substituting in the lower order equations. Consider, for example, the addition of a fourth order term to the Schrödinger-like equation (3.6)

$$
-\psi^{\prime \prime}(\hat{\rho})+\hat{U}(\hat{\rho}) \psi(\hat{\rho})=\lambda \psi^{(4)}(\hat{\rho}) .
$$

We now rewrite this as $\psi^{\prime \prime}=U \psi-\lambda \psi^{(4)}$ and take two derivatives to obtain $\psi^{(4)}=$ $(U \psi)^{\prime \prime}-\lambda \psi^{(6)}$. Substituting this in the right-hand side of (6.2) and working only to linear order in $\lambda$ then reduces the equation to second order

$$
-\psi^{\prime \prime}(\hat{\rho})+\hat{U}(\hat{\rho}) \psi(\hat{\rho})-\lambda(\hat{U}(\hat{\rho}) \psi(\hat{\rho}))^{\prime \prime}=\mathcal{O}\left(\lambda^{2}\right) .
$$

While this equation is no longer in manifest Schrödinger form, it can be so transformed if desired. Thus our analysis is in fact applicable to this more general case as well.

As we discussed at the end of section 4, the perturbative expansion of the spectral function in terms of higher derivative coefficients $\lambda_{i, j}$ strictly speaking only makes sense if these coefficients are chosen such that no additional turning points are introduced deep in the bulk. However, we argued that our formal perturbation series can still be used even in the case of higher derivatives with "wrong" sign, i.e. for the case where the effective potential bends upwards at large $\rho$. It would be interesting to determine if in a realistic theory, there are constraints on the signs of the coefficients $\lambda_{i, j}$, for example due to bulk causality or unitarity. It would also be interesting to study string theory embeddings of Lifshitz spacetimes, where the coefficients of higher derivative corrections can be determined exactly, and calculate the corrections to holographic correlation functions.

\section{Acknowledgments}

This work was supported in part by the US Department of Energy under grant DESC0007859. The work of CK is supported in part by the Danish Council for Independent 
Research project "New horizons in particle and condensed matter physics from black holes". KS is supported by the US National Science Foundation under grant NSF-PHY-1402971.

\section{A Perturbative expansion of the WKB integral}

We would like to obtain an approximate expression for the WKB integral (4.3) for a potential of the form

$$
\hat{U}(\hat{\rho})=\hat{U}_{0}(\hat{\rho})+\delta \hat{U}(\hat{\rho})
$$

where

$$
\hat{U}_{0}=\frac{\nu^{2}}{\hat{\rho}^{2}}+\frac{1}{\hat{\rho}^{2-2 / z}}-\hat{\omega}^{2},
$$

and $\delta \hat{U}$ represents a small correction to the potential. To be precise, we assume that $\delta \hat{U}$ is subdominant compared to the other terms in the potential for all $\hat{\rho}$ between the boundary and the classical turning point. To guarantee this, it is sufficient to demand that

$$
\delta \hat{U}(\hat{\rho}) \ll \hat{\omega}^{2} \quad \text { for } \quad 0 \leq \hat{\rho} \leq \hat{\rho}_{0} .
$$

We can then expand the turning point as follows:

$$
\hat{\rho}_{0}(t)=\hat{\rho}_{0}^{(0)}(1+t+\cdots),
$$

where

$$
t \sim \frac{\delta \hat{U}\left(\hat{\rho}_{0}^{(0)}\right)}{\hat{\omega}^{2}} \ll 1
$$

and $\hat{\rho}_{0}^{(0)}$ is the turning point of the unperturbed potential, i.e. $\hat{U}_{0}\left(\hat{\rho}_{0}^{(0)}\right)=0$. The relative size of $\delta \hat{U}$ at the unperturbed turning point is what controls the higher derivative expansion. The WKB integral (4.3) can be written as $S=S^{(0)}+\delta S$, where

$$
\delta S=S-S^{(0)}=\int_{\epsilon}^{\hat{\rho}_{0}(t)} d \hat{\rho} \sqrt{\hat{U}_{0}(\hat{\rho})+\delta \hat{U}(\hat{\rho})}-\int_{\epsilon}^{\hat{\rho}_{0}^{(0)}} d \hat{\rho} \sqrt{\hat{U}_{0}(\hat{\rho})} .
$$

One could attempt to simply expand the above expression formally in $t$ and $\delta \hat{U}$, and it turns out that this does indeed give the correct result (4.7). However, this approach is problematic, since $\hat{U}_{0}$ goes to zero at $\hat{\rho}_{0}^{(0)}$, and thus the formal expansion parameter $\delta \hat{U} / \hat{U}_{0}$ blows up at this location. The solution is to split up the integrals in (A.6) in a way that the integrand always has a well-defined expansion in terms of $\delta \hat{U}$. To do this, we shift the first integral by rescaling $x \equiv \hat{\rho} \hat{\rho}_{0}^{(0)} / \hat{\rho}_{0}(t)$, so that the upper bounds of both integrals are identical. We can then combine both terms to obtain

$$
\delta S \approx \int_{\frac{\epsilon}{1+t}}^{\epsilon} d x \sqrt{\frac{\nu^{2}}{x^{2}}+\frac{1+\frac{2}{z} t}{x^{2-2 / z}}-(1+2 t)+\delta \hat{U}(x)}+\int_{\epsilon}^{\hat{\rho}_{0}^{(0)}} d x \sqrt{\hat{U}_{0}(x)}[\sqrt{1+V(x)}-1],
$$

where

$$
V(x)=\frac{\delta \hat{U}(x)+\frac{2}{z \hat{\rho}^{2-2 / z}} t-2 t}{\hat{U}_{0}(x)}
$$


and we expanded to linear order in $t$. The first term in (A.7) is due to the shift of the lower bound of the first integral in (A.6). Assuming that $\lim _{x \rightarrow 0} x^{2} \delta \hat{U}(x)=0$, this term evaluates to $\nu t$ after we send $\epsilon \rightarrow 0$. To compute the second integral, notice that although $\hat{U}_{0}(x)$ itself blows up at the upper bound, the ratio $V(x)$ remains finite everywhere. Moreover, it is small by assumption, so we can expand (A.7) in terms of $V(x)$ :

$$
\delta S \approx \nu t+\int_{\epsilon}^{\hat{\rho}_{0}^{(0)}} d x \frac{\delta \hat{U}(x)+\frac{2}{z \hat{\rho}^{2-2 / z}} t-2 t}{2 \sqrt{\hat{U}_{0}}} .
$$

The integral over the terms linear in $t$ exactly cancels the $\nu t$ term, and we arrive at the final result:

$$
\delta S \approx \int_{\epsilon}^{\hat{\rho}_{0}^{(0)}} d x \frac{\delta \hat{U}(x)}{2 \sqrt{\hat{U}_{0}}} .
$$

This is the first order correction to the WKB integral in the presence of a perturbation $\delta \hat{U}$.

\section{B The free quadratic band crossing theory}

In the absence of interactions $(g=0)$, the action for the quadratic band crossing model, shown in (5.4), describes two species of free fermions with dispersion relations

$$
\epsilon_{ \pm}(\vec{k})=\left(t_{0} \pm \sqrt{\frac{\left(t_{1}^{2}+t_{2}^{2}\right)+\left(t_{1}^{2}-t_{2}^{2}\right) \cos 4 \theta_{k}}{2}}\right) k^{2},
$$

where $\epsilon_{+}(\vec{k})$ and $\epsilon_{-}(\vec{k})$ are the energies for the two species of fermions at momentum $\vec{k}$ respectively. The angle $\theta_{k}$ is the azimuthal angle of $\vec{k}$.

In contrast with the $2+1$ dimensional Dirac theory, where the $\mathrm{SO}(2)$ spatial rotational symmetry arises automatically (even if the speed of light is different along $x$ and $y$, the rotational symmetry can be obtained by rescaling), the $z=2$ model here in general only preserves a four-fold rotational symmetry (see the cosine term in the dispersion relation). Continuous rotational symmetry is only recovered at $t_{1}=t_{2}[12]$.

For $\left|t_{0}\right|<\left|t_{1}\right|$ and $\left|t_{0}\right|<\left|t_{2}\right|$, it is easy to realize that $\epsilon_{+}>0$ and $\epsilon_{-}<0$. Therefore, fermions with energy $\epsilon_{+}$and $\epsilon_{-}$are particles and holes respectively (or say particles and anti-particles). These particles and holes in general do not preserve the symmetry of charge conjugation, because $\epsilon_{+} \neq-\epsilon_{-}$, except when $t_{0}=0$. Thus charge conjugation only becomes a symmetry at $t_{0}=0$.

If $\left|t_{0}\right|$ is larger than both $\left|t_{1}\right|$ and $\left|t_{2}\right|$, then $\epsilon_{+}$and $\epsilon_{-}$will have the same sign, so that they are both particles (or both holes). If $\left|t_{1}\right| \neq\left|t_{2}\right|$ and $\left|t_{0}\right|$ takes a value between $\left|t_{1}\right|$ and $\left|t_{2}\right|$, the system is anisotropic and along certain directions we have a particle branch and a hole branch, but along certain other directions, we have two hole branches (or two particle branches). There is one special case with $\left|t_{0}\right|=\left|t_{1}\right|=\left|t_{2}\right|$. Here, one of the fermions has zero energy for any momentum, and this is known as a flat band. For a flat band, higher order spatial derivatives become important and will in general lift the energy degeneracy. Because a flat band is typically associated with an infinite density of states, it 
is usually unstable (towards a certain symmetry breaking ground state) when interactions are introduced.

In the main text, we focus on the case with $t_{0}=0$ and $t_{1}=t_{2}=1$, but most conclusions remains valid as long as $\left|t_{0}\right|<\left|t_{1}\right|$ and $\left|t_{0}\right|<\left|t_{2}\right|$. For this special case, the dispersion relation reduces to $\epsilon_{ \pm}(\vec{k})= \pm k^{2}$, as given in (5.6).

Open Access. This article is distributed under the terms of the Creative Commons Attribution License (CC-BY 4.0), which permits any use, distribution and reproduction in any medium, provided the original author(s) and source are credited.

\section{References}

[1] S. Kachru, X. Liu and M. Mulligan, Gravity duals of Lifshitz-like fixed points, Phys. Rev. D 78 (2008) 106005 [arXiv:0808.1725] [INSPIRE].

[2] D.T. Son, Toward an AdS/cold atoms correspondence: A Geometric realization of the Schrödinger symmetry, Phys. Rev. D 78 (2008) 046003 [arXiv: 0804.3972] [InSPIRE].

[3] K. Balasubramanian and J. McGreevy, Gravity duals for non-relativistic CFTs, Phys. Rev. Lett. 101 (2008) 061601 [arXiv:0804.4053] [InSPIRE].

[4] A. Adams, K. Balasubramanian and J. McGreevy, Hot Spacetimes for Cold Atoms, JHEP 11 (2008) 059 [arXiv:0807.1111] [INSPIRE].

[5] C. Keeler, G. Knodel and J.T. Liu, Hidden horizons in non-relativistic AdS/CFT, JHEP 08 (2014) 024 [arXiv:1404.4877] [INSPIRE].

[6] S. Leichenauer and V. Rosenhaus, AdS black holes, the bulk-boundary dictionary and smearing functions, Phys. Rev. D 88 (2013) 026003 [arXiv:1304.6821] [INSPIRE].

[7] C. Keeler, G. Knodel and J.T. Liu, What do non-relativistic CFTs tell us about Lifshitz spacetimes?, JHEP 01 (2014) 062 [arXiv: 1308.5689] [INSPIRE].

[8] S.-J. Rey and V. Rosenhaus, Scanning Tunneling Macroscopy, Black Holes and AdS/CFT Bulk Locality, JHEP 07 (2014) 050 [arXiv: 1403.3943] [INSPIRE].

[9] J. Hartong and N.A. Obers, Horava-Lifshitz Gravity From Dynamical Newton-Cartan Geometry, arXiv:1504.0746.

[10] D.M. Hofman and B. Rollier, Warped Conformal Field Theory as Lower Spin Gravity, Nucl. Phys. B 897 (2015) 1 [arXiv: 1411.0672] [INSPIRE].

[11] T. Griffin, P. Hor̂ava and C.M. Melby-Thompson, Lifshitz Gravity for Lifshitz Holography, Phys. Rev. Lett. 110 (2013) 081602 [arXiv: 1211.4872] [INSPIRE].

[12] K. Sun, H. Yao, E. Fradkin and S. Kivelson, Topological insulators and nematic phases from spontaneous symmetry breaking in $2 d$ fermi systems with a quadratic band crossing, Phys. Rev. Lett. 103 (2009) 046811 [arXiv: 0905. 0907] [INSPIRE].

[13] E. Ardonne, P. Fendley and E. Fradkin, Topological order and conformal quantum critical points, Annals Phys. 310 (2004) 493 [cond-mat/0311466] [InSPIRE].

[14] D.T. Son and A.O. Starinets, Minkowski space correlators in AdS/CFT correspondence: Recipe and applications, JHEP 09 (2002) 042 [hep-th/0205051] [INSPIRE]. 
[15] G. Policastro, D.T. Son and A.O. Starinets, The Shear viscosity of strongly coupled $N=4$ supersymmetric Yang-Mills plasma, Phys. Rev. Lett. 87 (2001) 081601 [hep-th/0104066] [INSPIRE].

[16] G. Policastro, D.T. Son and A.O. Starinets, From AdS/CFT correspondence to hydrodynamics, JHEP 09 (2002) 043 [hep-th/0205052] [INSPIRE].

[17] G. Policastro, D.T. Son and A.O. Starinets, From AdS/CFT correspondence to hydrodynamics. 2. Sound waves, JHEP 12 (2002) 054 [hep-th/0210220] [INSPIRE].

[18] C.P. Herzog, The Hydrodynamics of M-theory, JHEP 12 (2002) 026 [hep-th/0210126] [INSPIRE].

[19] C.P. Herzog, The Sound of M-theory, Phys. Rev. D 68 (2003) 024013 [hep-th/0302086] [INSPIRE].

[20] P. Kovtun, D.T. Son and A.O. Starinets, Viscosity in strongly interacting quantum field theories from black hole physics, Phys. Rev. Lett. 94 (2005) 111601 [hep-th/0405231] [INSPIRE].

[21] A. Buchel, J.T. Liu and A.O. Starinets, Coupling constant dependence of the shear viscosity in $N=4$ supersymmetric Yang-Mills theory, Nucl. Phys. B 707 (2005) 56 [hep-th/0406264] [INSPIRE].

[22] D.T. Son and A.O. Starinets, Viscosity, Black Holes and Quantum Field Theory, Ann. Rev. Nucl. Part. Sci. 57 (2007) 95 [arXiv: 0704.0240] [INSPIRE].

[23] J. Nilsson, A.H. Castro Neto, F. Guinea and N.M.R. Peres, Electronic properties of bilayer and multilayer graphene, Phys. Rev. B 78 (2008) 045405 [arXiv:0712.3259] [INSPIRE].

[24] A.H. Castro Neto, F. Guinea, N.M.R. Peres, K.S. Novoselov and A.K. Geim, The electronic properties of graphene, Rev. Mod. Phys. 81 (2009) 109 [arXiv:0709.1163] [INSPIRE].

[25] V.N. Kotov, B. Uchoa, V.M. Pereira, A.H.C. Neto and F. Guinea, Electron-Electron Interactions in Graphene: Current Status and Perspectives, Rev. Mod. Phys. 84 (2012) 1067 [arXiv: 1012.3484] [INSPIRE].

[26] K. Sun, W.V. Liu, A. Hemmerich and S.D. Sarma, Topological semimetal in a fermionic optical lattice, Nature Phys. 8 (2012) 67 [arXiv:1011.4301] [INSPIRE]. 